Research Article

\title{
Damage Identification by the Data Expansion and Substructuring Methods
}

\author{
Eun-Taik Lee ${ }^{1}$ and Hee-Chang Eun (D) $^{2}$ \\ ${ }^{1}$ Department of Architectural Engineering, Chung-Ang University, Seoul, Republic of Korea \\ ${ }^{2}$ Department of Architectural Engineering, Kangwon National University, Chuncheon, Republic of Korea
}

Correspondence should be addressed to Hee-Chang Eun; heechang@kangwon.ac.kr

Received 24 September 2018; Revised 29 October 2018; Accepted 18 November 2018; Published 11 December 2018

Guest Editor: Tayfun Dede

Copyright (c) 2018 Eun-Taik Lee and Hee-Chang Eun. This is an open access article distributed under the Creative Commons Attribution License, which permits unrestricted use, distribution, and reproduction in any medium, provided the original work is properly cited.

\begin{abstract}
Structural damage can be detected by comparing the responses before and after the damage. The responses are transformed into curvature, strain, and stress, among others, which characterize the mechanical behavior of the structural members, and can be utilized as damage indices for damage detection. The damage of a truss structure can rarely be detected by the displacements only at nodes. This work investigates damage detection methods using the stress or stiffness variation rate of the truss element before and after the damage. This paper considers three different cases according to the number of measurement locations. If the complete responses at a full set of degrees of freedom are measured, the stiffness variation rates of the elements are calculated accurately, and the damage can be explicitly detected despite external noise. If the number of measured data points is fewer than the system order, the displacements are estimated by the data expansion method, and the damage-expected regions are predicted by the stiffness variation rates. Apart from the explicitly damaged elements, the substructuring approach is adopted for closer damage detection with several measurement sensors despite external noise. It is illustrated by the examples that three cases are compared numerically. The numerical examples compare and analyze the numerical results of the three cases.
\end{abstract}

\section{Introduction}

Structural maintenance has received significant attention in the construction industry. Existing structures have been diagnosed, repaired, reformed, and remodeled for improving the structural performance and durability. Structural health monitoring and nondestructive tests for evaluating structural performance have been developed with the advent of new measurement sensors and technologies, and techniques for structural health monitoring have been applied in the field.

The health state of a structure is evaluated by the response, internal strain, or internal stress, among others. Structural defects are detected in the process by comparing the responses before and after the structural performance deterioration. The measured responses are transformed into curvature, strain, and stress for damage detection. The measured displacements in the truss structure should be transformed into axial stresses for evaluating the existence of damage.
It is impractical to collect the responses at a full set of degrees of freedom (DOFs) because of the limited number of sensors. The number of measurement sensors is practically fewer than the system order. Thus, incomplete measurement data should be expanded, or the system order should be reduced.

The damage identification method has become a prominent research field. The identification of a global structure requires a large number of sensors and unknowns; however, the substructuring method needs only several sensors for response measurement.

Blachowski et al. [1] presented a damage quantification method for the truss structure without identifying modal parameters or solving a global optimization problem. The method quantifies the damage in a given substructure with only a small subset of sensors. Park and Reich [2] provided two complementary methods for model-based structural damage detection, based on changes in the localized flexibility and invariance properties of the elemental or 
substructural transmission zeros. Xing and Mita [3] proposed a substructure approach for identifying localized damage on each substructure using only three sensors. Ugalde et al. [4] presented a substructuring approach to predict the health of each substructure by means of the vector autoregressive variation with the exogenous model. Hou et al. [5] proposed a substructure damage identification method using local free responses. Estimating additional unknown inputs by the algorithm, without measurements of the substructure interface DOFs, Lei et al. [6] applied the damage detection method by means of the degradation of the identified substructural element stiffness values. Lee and Eun [7] presented a modelbased substructuring method for local damage detection using the frequency response function measured at the interfaces between the adjacent substructures. Li et al. [8] proposed a substructural damage identification approach based on dynamic response reconstruction and a dynamic response sensitivity-based method.

An et al. [9] presented a stochastic damage-locating vector method as a useful tool for damage localization in steel truss bridges. An et al. [10] proposed a damage localization approach for truss structures using the curvature difference method of strain waveform fractal dimensions. Yang and Jin [11] presented a damage detection method for truss structures using incompletely measured modes, with the aid of the eigenvalue perturbation method. Kaven and Zolghadr [12] proposed a damage detection method using the differences between structure characteristics, such as natural frequencies and mode shapes of truss structures. $\mathrm{Xu}$ and $\mathrm{Wu}$ [13] developed a damage detection technique based on strain data under ambient excitation using the environmental excitation incomplete strain mode. Artar and Daloğlu [14] presented a damage identification method to update the numerical model and to predict damage location and severity in the space frame using genetic algorithms.

This study considers methods for predicting damaged elements depending on complete and incomplete measurement data, including the noise effect. The measured and estimated displacements are transformed into the internal stress of the elements, and the stiffness variation rate is used as a damage index. By dividing a full truss structure into several damage-expected substructures, this work proposes the substructuring method to estimate damaged elements. This method offers merits in reducing the model order and number of measurement locations by the static-based and dynamic-based data expansion method [15] and is insensitive to external noise. The damage detection methods using complete and incomplete measurements are investigated in numerical examples. The validity of the proposed static-based and dynamic-based substructuring method is illustrated in examples, depending on external noise.

\section{Substructuring Method for Damage Detection}

Structural diagnosis is conducted by means of the comparison of the response, strain, stress, and stiffness in healthy and unhealthy states, or the measurement data only in the damage-expected state without the baseline data. This method is performed by a response comparison in both states. This work compares three damage detection methods, depending on the number of measurement locations to be causable in the structural performance evaluation. The three different cases are as follows: (1) damage detection using complete measurement data; (2) damage detection using the data to expand fewer measurement data than the model order; and (3) damage detection by measurement data and data expansion at the substructures to divide an entire structure. Moreover, the effect of the noise contained in the measurement data is examined. Figure 1 summarizes the flow of this study to detect damaged elements.

\subsection{Static Approach}

2.1.1. Complete Measurement Data. The equilibrium equation of a finite element model with $n$ DOFs in the intact state can be expressed by

$$
\mathbf{F}=\widehat{\mathbf{K}} \widehat{\mathbf{u}},
$$

where $\widehat{\mathbf{K}}$ and $\widehat{\mathbf{u}}$ denote the $n \times n$ positive definite stiffness matrix and $n \times 1$ displacement vector in the healthy state, respectively. Moreover, $\mathbf{F}$ is the $n \times 1$ external force vector. The displacement data at a full set of DOFs may be measured by measurement sensors or calculated by the finite element model for damage detection.

The truss structure is composed of axial members for carrying the axial load, and each member has axial displacement DOFs at both end nodes. The measured displacements are transformed into the axial stress related to the axial stiffness, and the damage can be detected by comparing the stresses or stiffness. This complete method exhibits the limitation of requiring the same number of measurement sensors as the system order.

2.1.2. Incomplete Measurement Data. It is not practical to measure the responses for the entire DOFs because of fewer measurement sensors than the system order. This indicates that the full set of displacements should be estimated by means of data expansion. Assuming that the static responses are taken as $m(m<n)$ DOFs, constraints must be implemented to describe the static behavior of the entire system. These can be written as

$$
\mathbf{A u}=\mathbf{b},
$$

where the $m \times n$ coefficient matrix $\mathbf{A}$ is a Boolean matrix for defining the measurement locations and the vectors $\mathbf{u}$ and $\mathbf{b}$ are the $n \times 1$ actual displacement vector and $m \times 1$ measured response vector, respectively.

Constraint forces for pulling the unconstrained path of the initial system into the constrained path are required. Combining equations (1) and (2), reference [15] derives the constrained equilibrium equation as follows:

$$
\mathbf{u}=\widehat{\mathbf{u}}+\widehat{\mathbf{K}}^{-1 / 2}\left(\mathbf{A} \widehat{\mathbf{K}}^{-1 / 2}\right)^{+}(\mathbf{b}-\mathbf{A} \widehat{\mathbf{u}})
$$

where "+" denotes the Moore-Penrose inverse. The displacements corresponding to the entire DOFs are estimated 


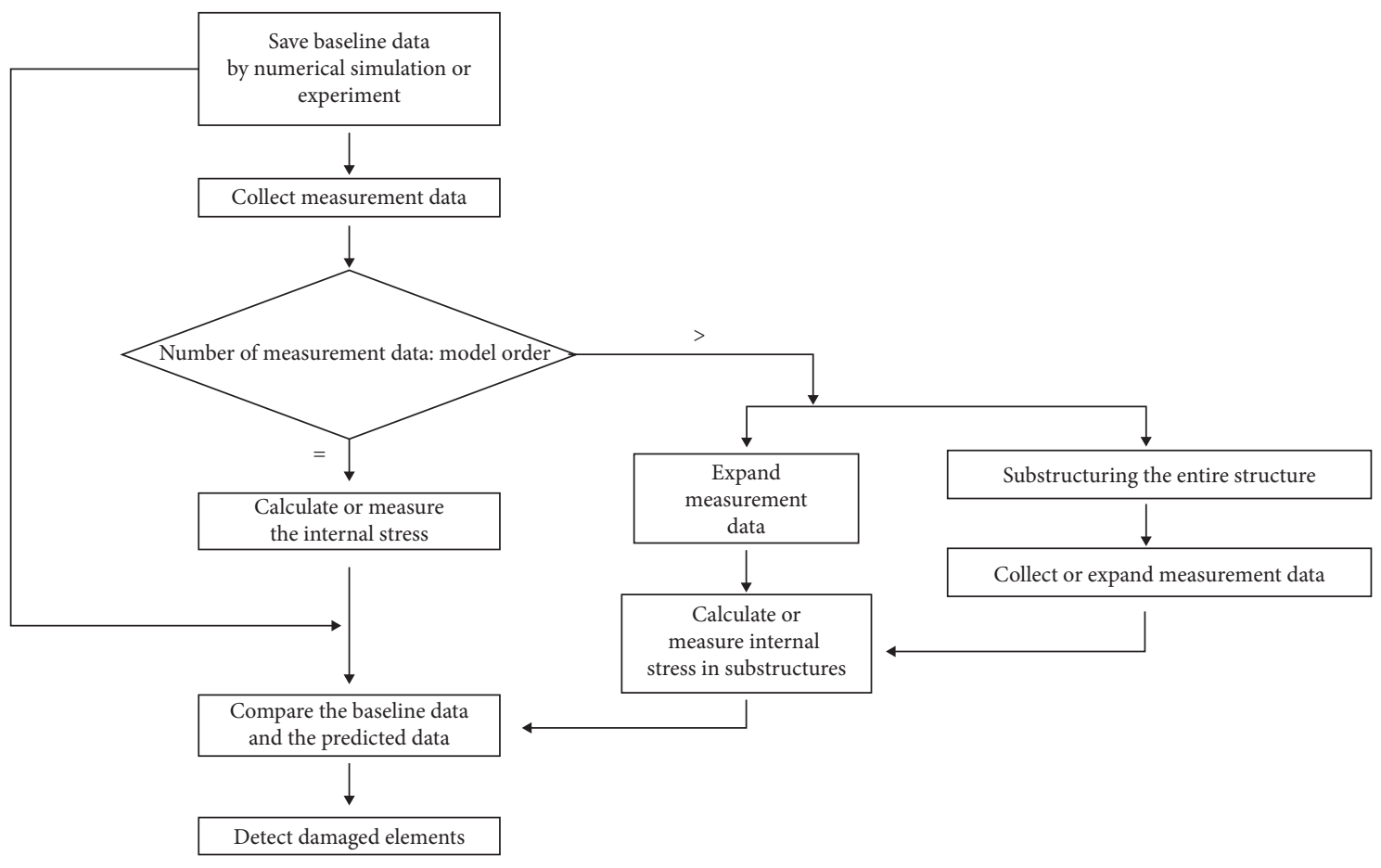

Figure 1: Flow chart of this work to detect damaged elements.

by using equation (3), satisfying the constraints of equation (2). The second term on the right-hand side of equation (3) represents the displacement variation deviating from the initially established trajectory. Moreover, by premultiplying the second term on the right-hand side of equation (3) by $\widehat{\mathbf{K}}$, the constraint force vector $\mathbf{F}^{\mathfrak{c}}$ is obtained by

$$
\mathbf{F}^{\mathfrak{c}}=\widehat{\mathbf{K}}^{1 / 2}\left(\mathbf{A} \widehat{\mathbf{K}}^{-1 / 2}\right)^{+}(\mathbf{b}-\mathbf{A} \widehat{\mathbf{u}}) \text {. }
$$

The internal stresses of the truss structure can be calculated accurately by using the displacements and axial stiffness. However, the displacements for expanding the incomplete measurements rarely coincide with the accurate displacements. Moreover, the calculated internal stresses of the truss elements do not coincide with the exact values. The following example investigates the feasibility of the damage detection by comparing the internal stresses estimated by the expanded data with the initially established stresses.

Example 1. The adequacy of the estimated responses using equation (3) is investigated in the numerical example of a plane truss structure, as illustrated in Figure 2. In the figure, the nodal points and members are numbered. Each node has two DOFs of the horizontal and vertical responses $u$ and $v$, respectively. The truss is composed of six nodes, nine members, and nine DOFs, except for boundary conditions. All members have the same elastic modulus of $200 \mathrm{GPa}$ and cross-sectional area of $2.5 \times 10^{-3} \mathrm{~m}^{2}$. The simply supported truss has a single span. Its length is $12 \mathrm{~m}$, its height is $3 \mathrm{~m}$, and each bay is $4 \mathrm{~m}$ long. The responses in the intact state can be calculated by the finite element method under the action of the external force of $10 \mathrm{~N}$ in the downward direction of

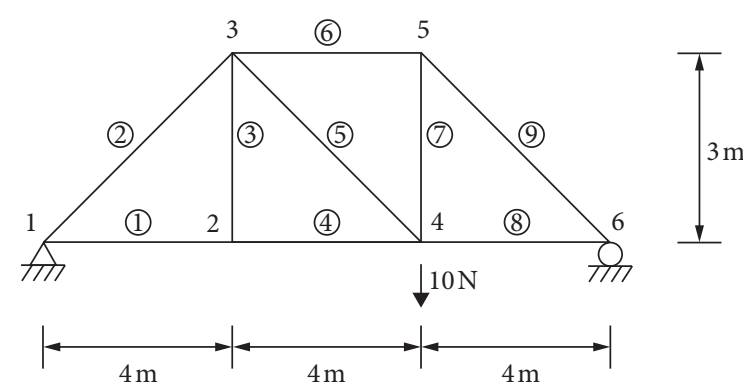

Figure 2: A three-bay truss structure.

node 4 . The elemental stiffness matrices and responses in the initial state should be saved for subsequent analysis.

The truss exhibits a $20 \%$ section loss at element (4) and $30 \%$ section loss at element (8). The vertical displacements $v_{2}$, $v_{3}, v_{4}$, and $v_{5}$, numerically simulated in the damaged state, were utilized as measurement data. Inserting the equilibrium equation in the intact state and measurement data in the damaged state into equation (3), the displacements for the entire DOFs are estimated. The truss structure is composed of axial members and pin joints. Thus, unlike the beam, the truss structure represents discontinuous responses at the element nodes. The differences between the actual and estimated responses are displayed in Figure 3. The plots illustrate that the resulting responses coincide at measured DOFs, but a discrepancy of a very small magnitude is evident at the other DOFs. The expansion method does not describe the accurate responses, except for the constraint conditions. The utilization of inaccurately expanded data leads to incorrect analysis. 


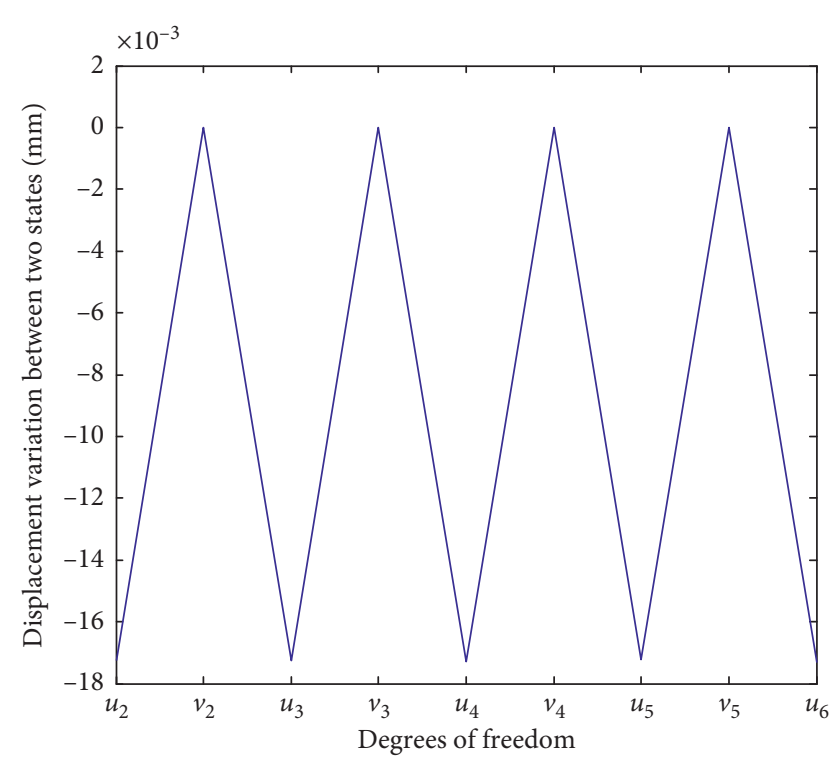

Figure 3: Difference between actual and estimated responses of the truss model.

The stress variation rate $\alpha$, defined as the stress variation with respect to the stress on an element in the damage state, can be calculated by

$$
\alpha=\frac{\sigma_{\mathrm{d}}-\sigma_{\mathrm{u}}}{\sigma_{\mathrm{d}}} \times 100(\%)
$$

where $\sigma_{\mathrm{u}}$ and $\sigma_{\mathrm{d}}$ denote the axial stresses in the undamaged and damaged states, respectively. Moreover, $\sigma_{\mathrm{d}}$ is calculated using the cross-sectional area and axial stiffness in the intact state and measurement data in the damaged state.

The stress variation rates in Figures 4(a) and 4(b) are obtained using the complete and incomplete measurement data, respectively. It is observed that accurate stress variation rates for the damaged elements can be obtained by using the complete measurement data. However, the plot using the incomplete measurement data exhibits very small variation rates, regardless of the damaged elements, and the damaged elements cannot be detected.

This expansion method exhibits the drawbacks of inaccurately estimating the expanded data and yielding inconsistent results depending on the sensor location and number. Thus, this work considers the analytical method for detecting the damage by dividing an entire structure into substructures in order to reduce the sensor number and detecting damage elements more reliably.

2.1.3. Substructuring Method. The limited number of sensors and unclearness of the damaged locations make a more detailed diagnosis method necessary. One precise diagnosis method may be the substructuring method, whereby an entire structure is divided into several substructures, as illustrated in Figure 5, and the damage is detected by measurements on each substructure. The damage detection in the substructure is carried out by means of a similar process to those of the previous complete and incomplete methods, depending on the number of measurement locations. The measured or estimated displacement data are transformed into the stress of the truss structure.

The damage yields the variation in the element stress, and it may be utilized as a damage index for evaluating the structural performance. Assuming a constant mass before and after the damage, the stress variation rate in equation (5) can be applied for detecting the damage. The stress variation is related to the cross-sectional variation under a constant axial force. The axial stiffness $k$ is also related to the variation in the cross-sectional area with the same elastic modulus and member length. Thus, equation (5) for the axial members can be expressed by

$$
\begin{aligned}
\alpha & =\left(\frac{\left(E\left(A_{\mathrm{d}}\right) / L\right)-\left(E\left(A_{\mathrm{u}}\right) / L\right)}{\left(E\left(A_{\mathrm{d}}\right) / L\right)}\right) \times 100(\%) \\
& =\left(\frac{k_{\mathrm{d}-} k_{\mathrm{u}}}{k_{\mathrm{d}}}\right) \times 100(\%),
\end{aligned}
$$

where $k_{\mathrm{d}}$ and $k_{\mathrm{u}}$ represent the stiffness values in the damaged and undamaged states, respectively, while $A_{\mathrm{d}}$ and $A_{\mathrm{u}}$ are the cross-sectional areas in the damaged and undamaged states, respectively. It is observed that the stiffness variation of equation (6) has the same meaning as the stress variation of equation (5).

2.2. Dynamic Approach. In the dynamic approach, mode shape data are utilized as measurement data as with the static displacements. The measurement data may be a full set of displacement DOFs or the data expanded from incomplete measurements. The stress variation rates in the truss elements are calculated by equation (5), or the stiffness variation rate is predicted by equation (6). The damage is detected by the stiffness variation rate plots.

Example 2. The above methods are examined in the numerical example in order to detect the damaged elements of the plane truss structure displayed in Figure 6. The numbering of nodes and members is indicated in the figure. Each node has two DOFs of the horizontal and vertical responses $u$ and $v$, respectively. The truss structure of 12 bays is simply supported and is composed of 24 nodes and 45 members. All members have the same elastic modulus of $200 \mathrm{GPa}$, cross-sectional area of $2.5 \times 10^{-3} \mathrm{~m}^{2}$, and density of $7,860 \mathrm{~kg} / \mathrm{m}^{3}$, as in the previous example. The total length of the truss is $6 \mathrm{~m}$, its height is $0.6 \mathrm{~m}$, and each bay is $0.5 \mathrm{~m}$ long. The truss is subjected to an external force of $10 \mathrm{~N}$ in the downward direction of node 7 . It has multiple damages of $20 \%$ section loss at elements 4,14 , and 42. The numerically simulated responses and stiffness values in the intact state are saved as baseline data for comparison with the numerical results in the damaged state. The above three static methods are compared, and one dynamic method is presented in this example. Moreover, each method evaluates the effect of the noise contained in the measured data. 


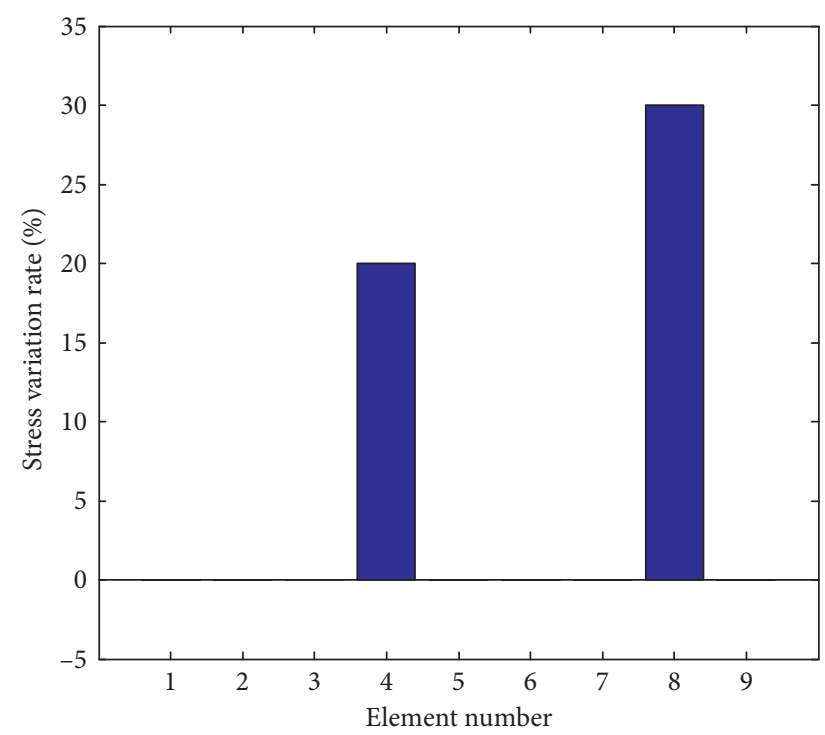

(a)

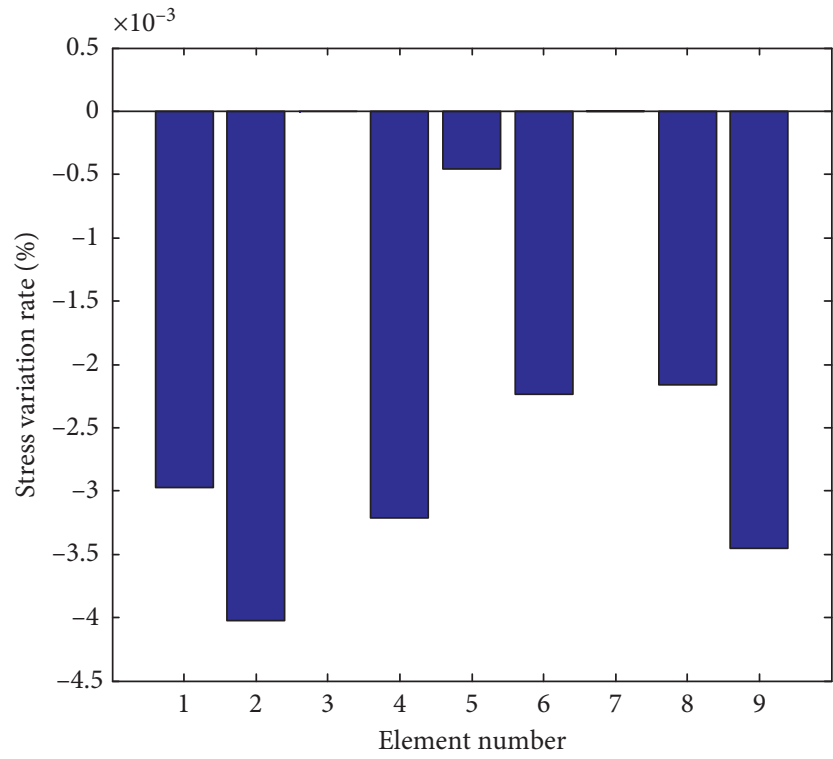

(b)

FIGURE 4: Stress variation rate of the truss model: (a) complete measurement data; (b) incomplete measurement data.

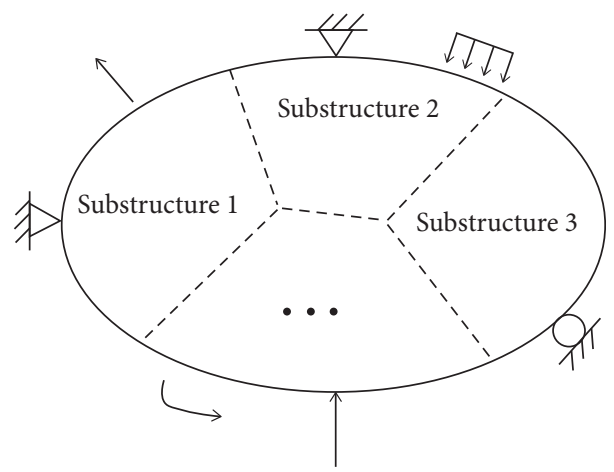

FIGURE 5: Substructuring of an entire structure.

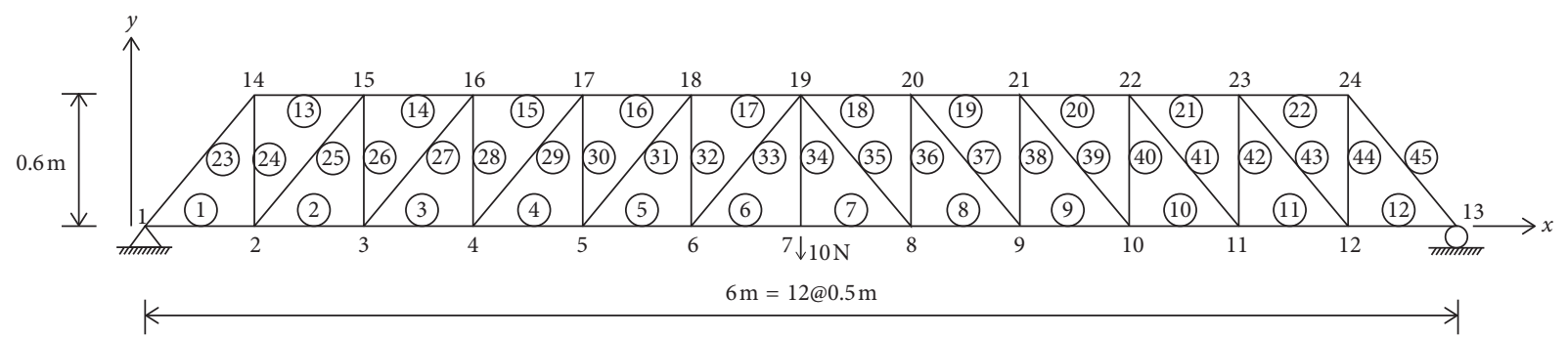

FIgURe 6: Numerical model of the truss structure for damage detection.

2.2.1. Complete Measurement Data. Figure 7 illustrates the numerical results calculated from the complete noise-free measurement data before and after the damage. Figure 7 (a) represents the horizontal and vertical displacement variations before and after the damage. The plots display the abrupt changes at the node to move from the lower to the upper chords. The damage positions at the element represent the abrupt displacement variation owing to the stiffness deterioration. However, the continuous displacement plots at the lower and upper chords do not provide any damage information without any additional considerations, such as flexural curvature. Figure 7(b) represents the stiffness variation rates calculated by equation (6), respectively. It is observed that abrupt stiffness variations are located at the damaged elements, and the variation rate coincides with the damage degree of $20 \%$. The application indicates that the damage can be clearly detected if accurate information is provided on the completely measured displacements. 


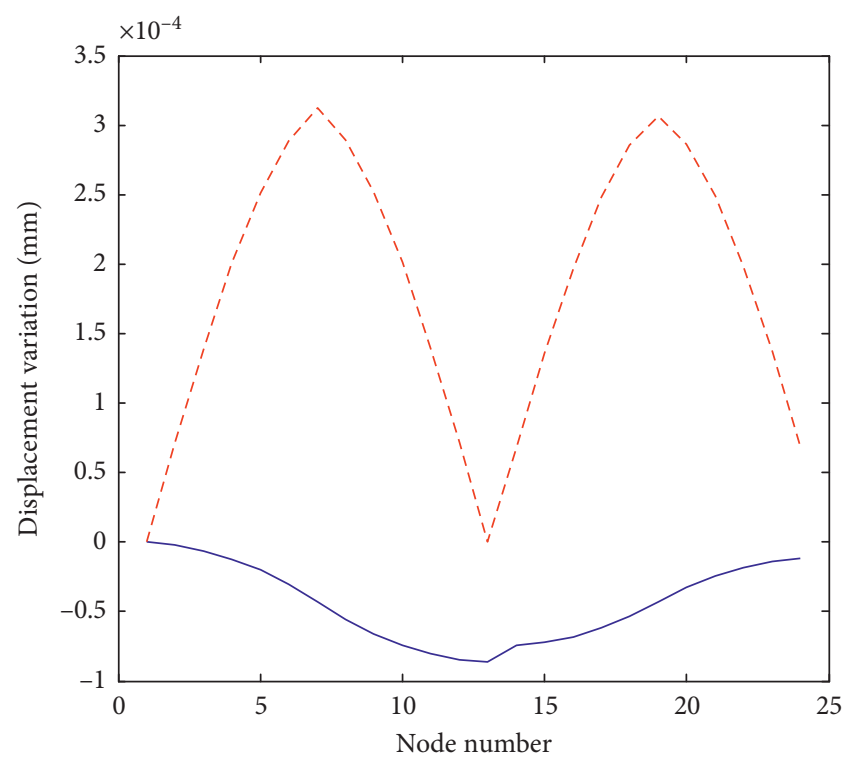

(a)

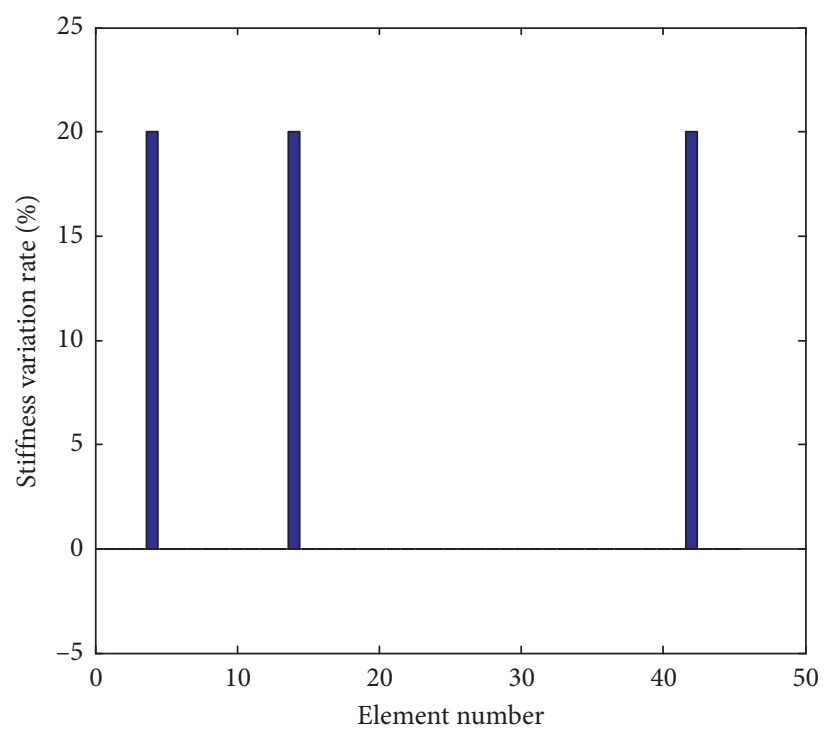

(b)

Figure 7: Numerical results using the complete noise-free measurement data: (a) displacement variations; (b) stiffness variation rate. In (a), the solid line indicates the horizontal displacement and the dashed line indicates the vertical displacement.

The presence of external noise leads to a slight variation in the displacements, deviating from the accurate trajectory. The measurement data contain errors contaminated by external noise. The $i$-th simulated or measurement dataset $\psi_{i}$ is established as

$$
\psi_{i}=\psi_{0, i}\left(1+\gamma \xi_{i}\right)
$$

where $\gamma$ denotes the relative magnitude of the error, $\xi_{i}$ is a random number variant in the range of $[-1,1]$, and $\psi_{0, i}$ is the $i$-th noise-free dataset. The applicability of the proposed method is investigated in the numerical example.

Figure 8(a) represents the displacement curves containing $1 \%$ noise. In order to reduce the effect owing to external noise, the mean values of 10 repeated numerical results were utilized as the measurement data for evaluating the validity of the method. It is observed that the plots in Figure 8(a) represent the displacement variations, do not indicate any peculiarity related to the damage, and exhibit difficulties in detecting the damage. Figure 8(b) displays the stiffness variation rate plot. The noise leads to irregular stiffness variations, unlike in the noise-free case. The damaged elements are clearly detected by the plots. The stiffness variation rates do not coincide with the actual damage degree owing to the noise. This example indicates that accurate damage detection is possible when accurate measurement data despite external noise are provided. It is demonstrated that the stiffness variation rate plots are almost the same except for the irregularities owing to noise.

2.2.2. Incomplete Measurement Data. At this time, we consider damage detection using measurement data with fewer DOFs than the system order. The vertical displacements at 22 nodes, except for support nodes 1 and 13, were measured, and the mean values of 10 repeated results were recorded. By inserting the numerically simulated measurement data and equilibrium equation in the intact state into equation (3), the displacements for the entire DOFs are estimated. Figure 9(a) illustrates the noise-free displacement differences before and after the damage. It is observed that the curve is nearly similar to that of Figure 7(a), using the complete measurement data. The damage cannot be detected from the plots. The estimated displacements are used to predict the internal stresses of the truss members. Figures 9(b)-9(f) illustrate the stiffness variation rates in the lower and upper chord members, diagonal members, and vertical members before and after the damage. Overall, the plots exhibit very small variation rates, except for the lower chord members and vertical members, as indicated in Figure 9. This is the reason that the expanded method rarely describes accurate responses, and the lower chord members are affected by the external load. The slash lines in Figure 10 represent the elements with high stiffness variation rates by the members. It is indicated that the damages are located at the left half of the midspan rather than at the right half, representing higher stiffness variation rates. Moreover, higher internal stress rates are represented at the left half of the midspan than at the right half. The highest stiffness variation rates are concentrated on the left-end support and loading point. The abrupt stiffness change in vertical member 42 is also illustrated. It is expected that the damaged members will be positioned at the left half region of the midspan and at vertical member 42 . Thus, the plots are merely used for estimation and do not clearly indicate the damaged elements. It can be concluded that this approach cannot detect the explicitly damaged elements.

This example also considers the noise effects included in the incomplete measurement data. The measurement data 


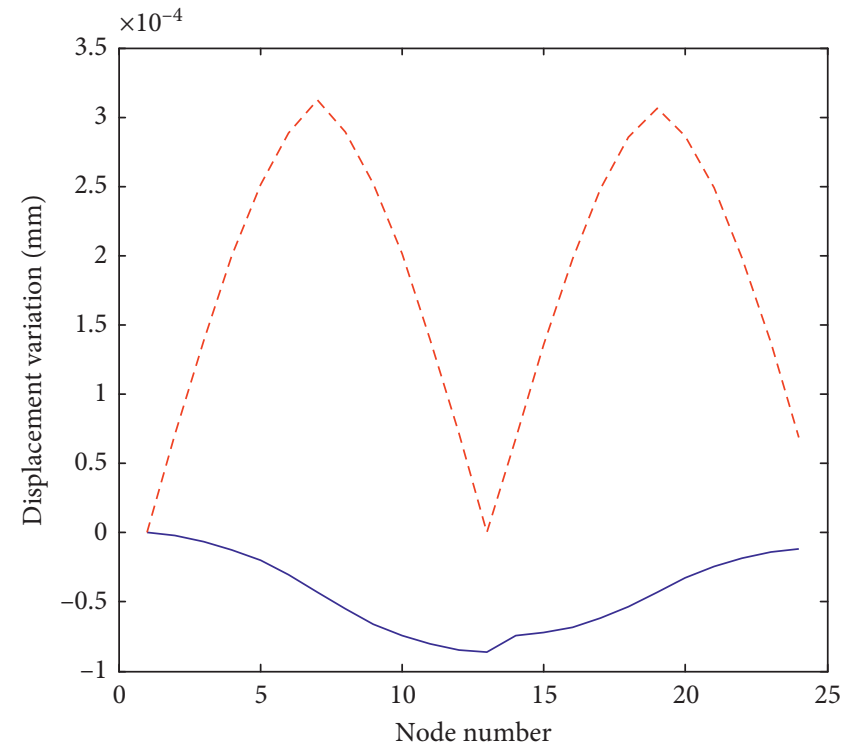

(a)

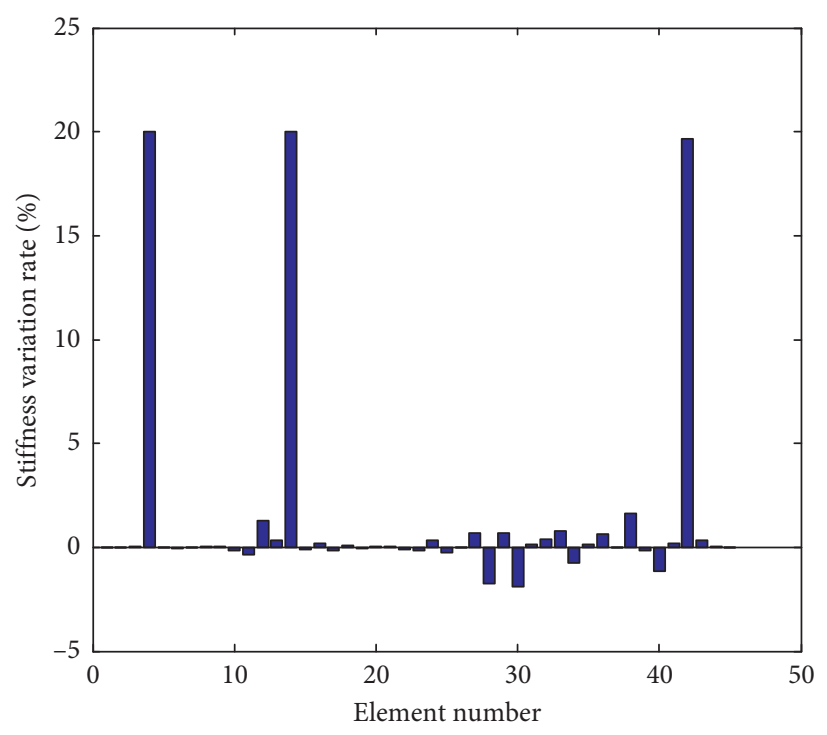

(b)

FIGURE 8: Numerical results using the complete measurement data contaminated by $1 \%$ noise level: (a) displacement variations; (b) stiffness variation rate. In (a), the solid line indicates the horizontal displacement and the dashed line indicates the vertical displacement.

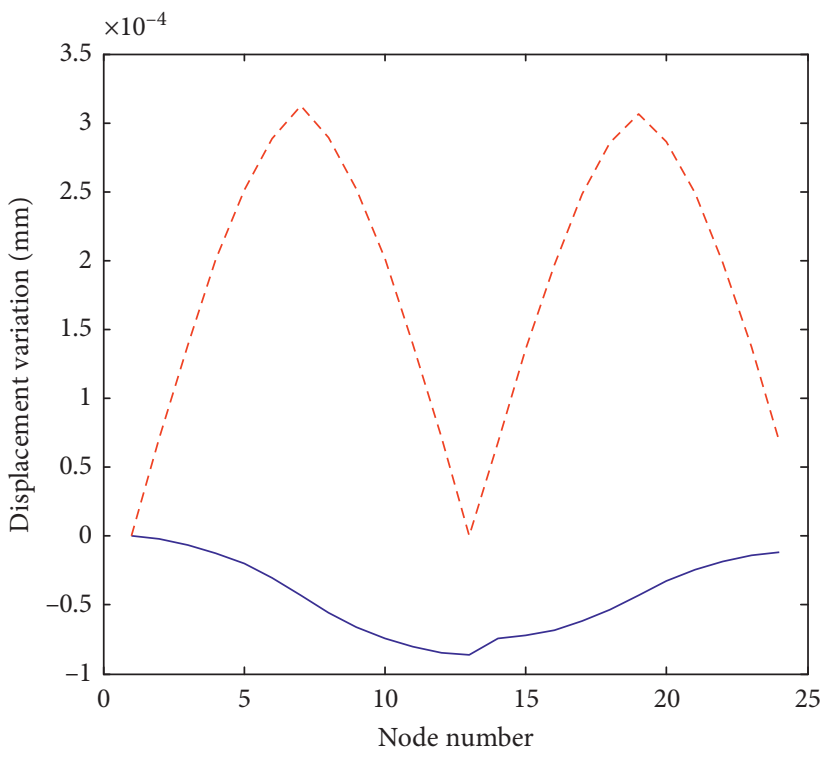

(a)

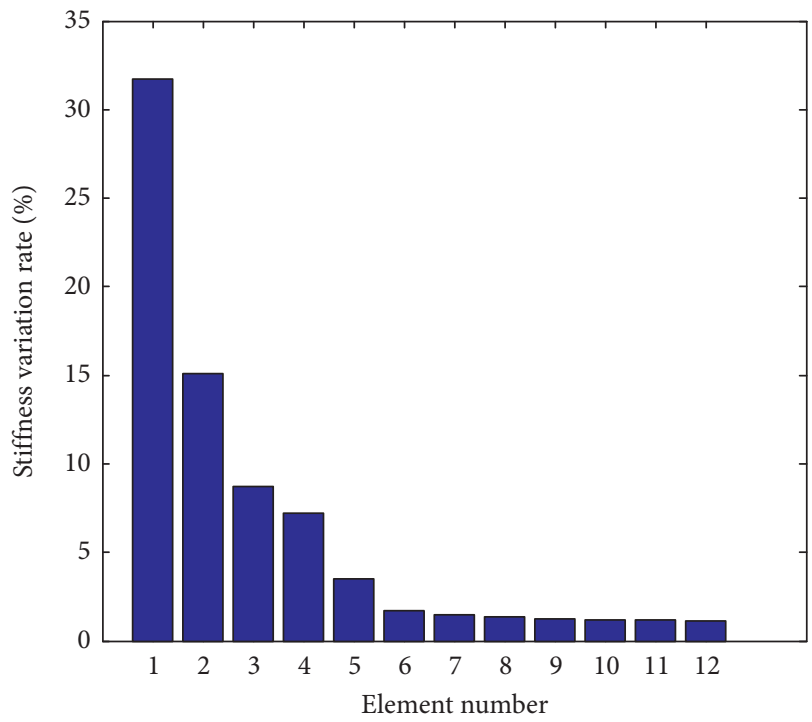

(b)

Figure 9: Continued. 


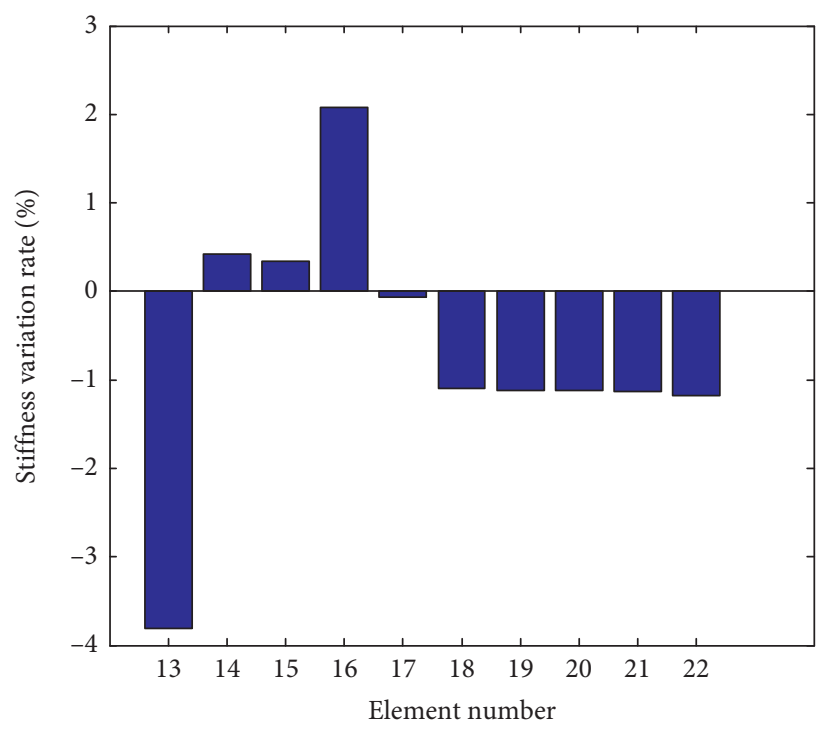

(c)

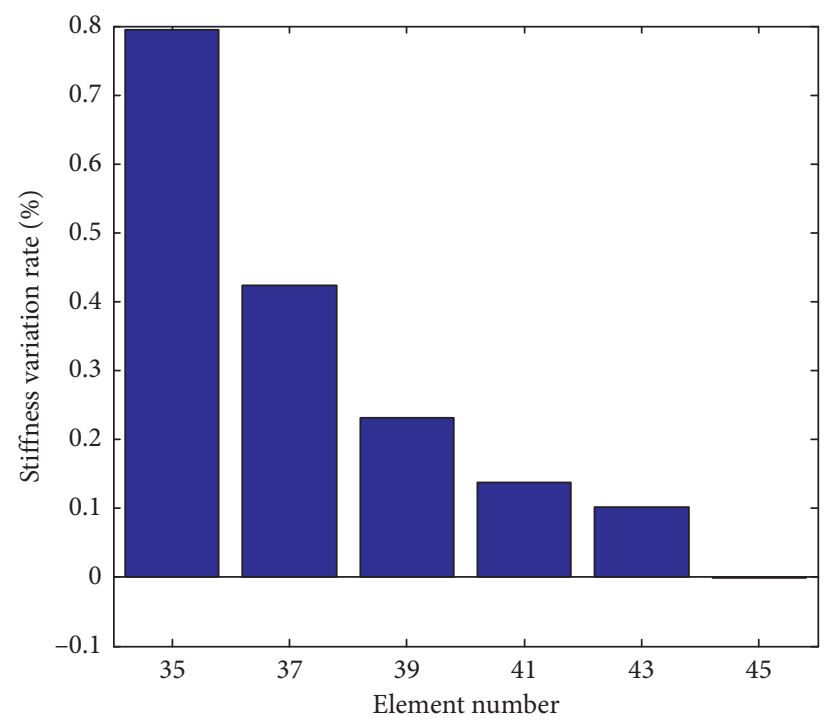

(e)

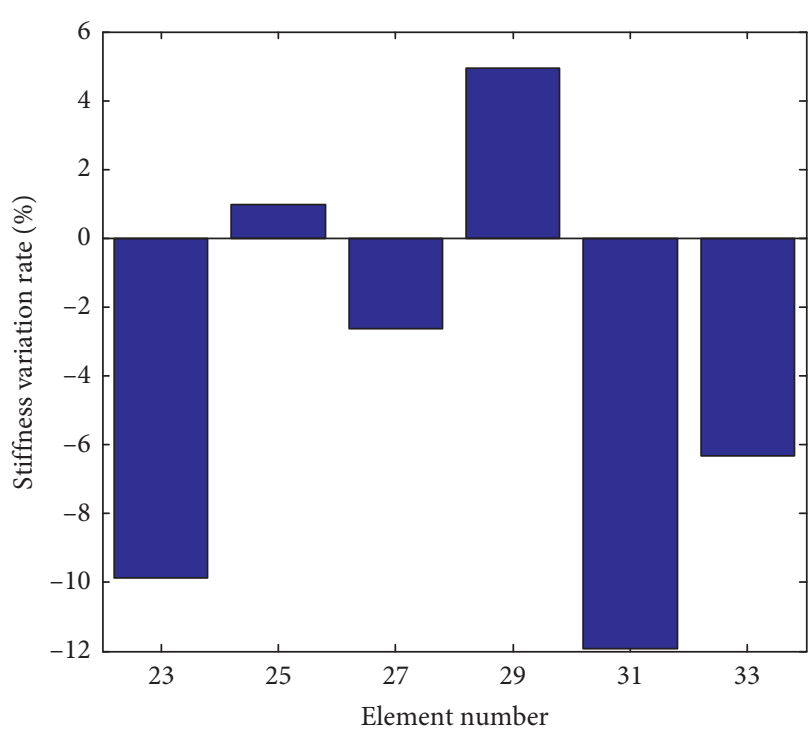

(d)

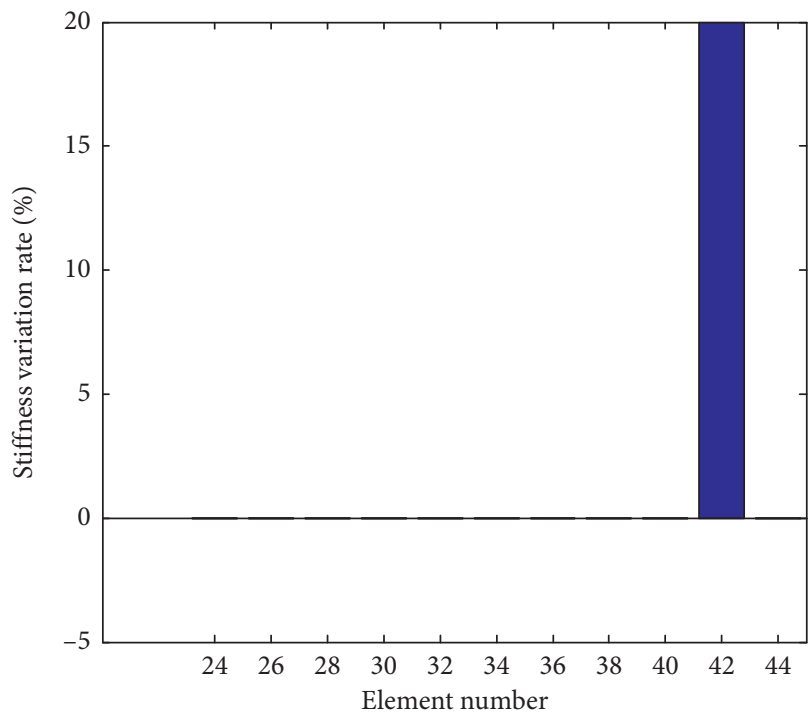

(f)

FIGURE 9: Damage detection using the expanded noise-free measurement data: (a) displacement variations; (b) stiffness variation rate at lower chords; (c) stiffness variation rate at upper chords; (d) stiffness variation rate at "/"-type diagonal elements; (e) stiffness variation rate at "I"-type diagonal elements; (f) stiffness variation rate at vertical elements. In (a), the solid line indicates the horizontal displacement and the dashed line indicates the vertical displacement.

are contaminated by $1 \%$ noise at the same DOFs as before and are expanded to the full set of DOFs. The numerical results are illustrated in Figure 11. The plots are very similar to those in Figure 9, and this method is not sensitive to external noise. Damage detection should be conducted by focusing on the region representing high stiffness variation rates. Thus, it is desirable to perform the analysis on the damage-expected element group with several measurement sensors, which constitutes the substructuring method.

2.2.3. Substructuring Method. We consider the damage detection of a substructure to be isolated from the truss structure illustrated in Figure 12. The substructure in Figure 12 is composed of 13 elements and eight nodes. Multiple damages are located at elements 5,17 , and 29 , with a $20 \%$ section loss. Measurements were taken at nine of 16 DOFs, as indicated in the figure. The number of sensors was significantly reduced, from 22 sensors in the previous test to nine in this test. The sensor locations are indicated in Figure 12. The mean values of the 10 repeated numerical experiments were taken for reducing the noise effect contained in the measurement data. The number of measurement locations is fewer than the substructure order, and data expansion is carried out.

Figure 13 displays the stiffness variation rate plots corresponding to the noise-free and 5\% noise cases. Both 


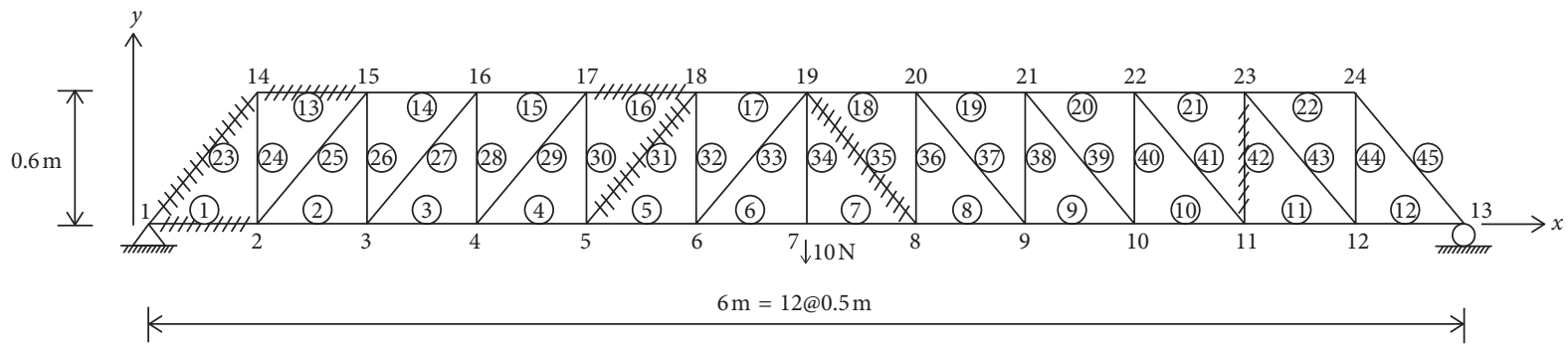

Figure 10: Truss members to represent high stress variation rates.

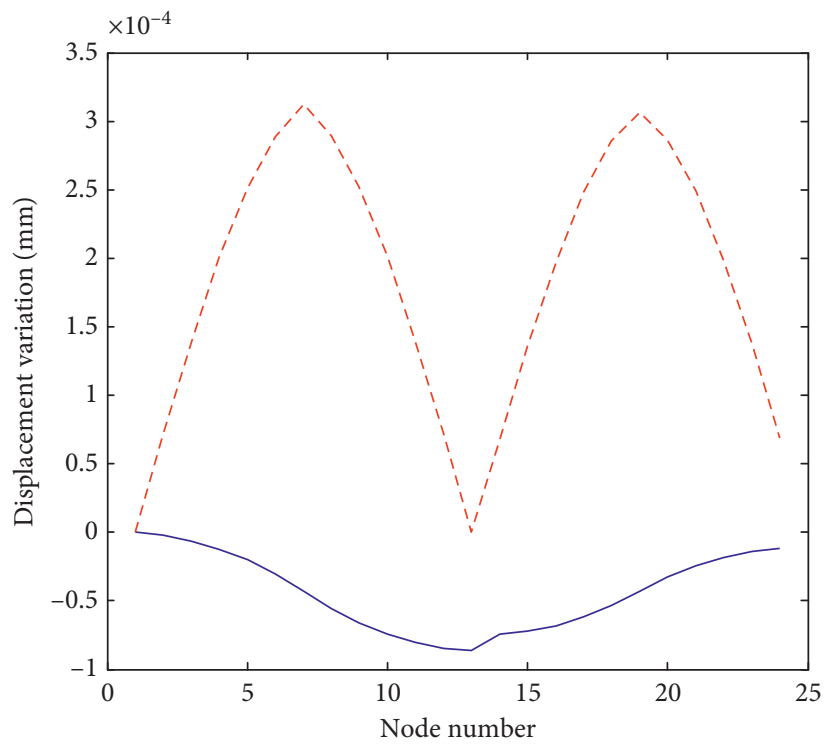

(a)

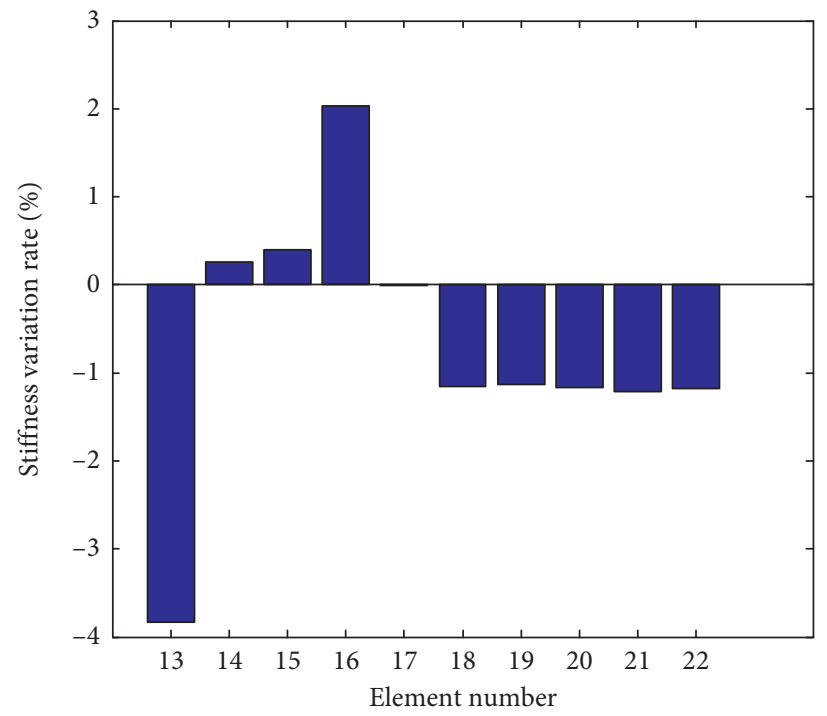

(c)

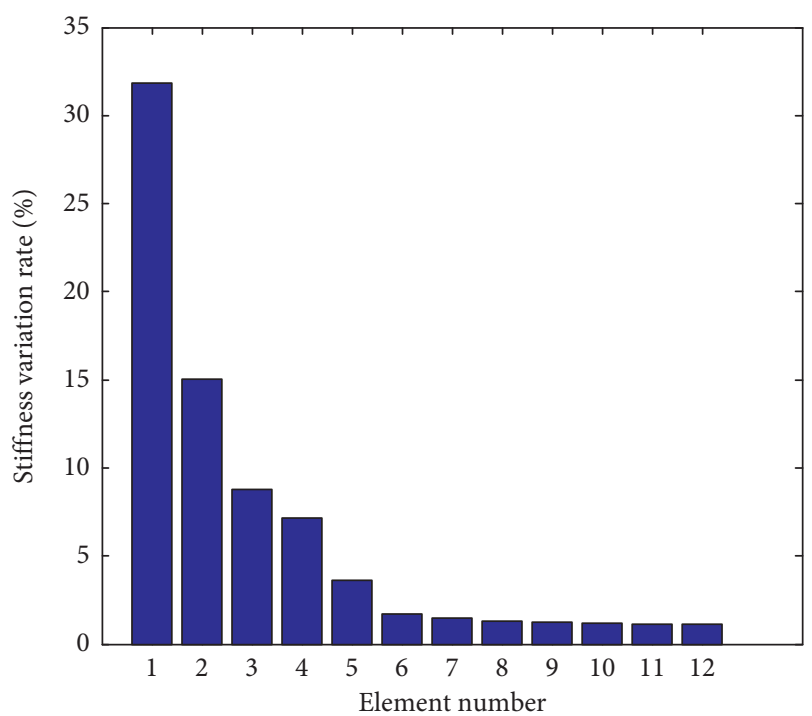

(b)

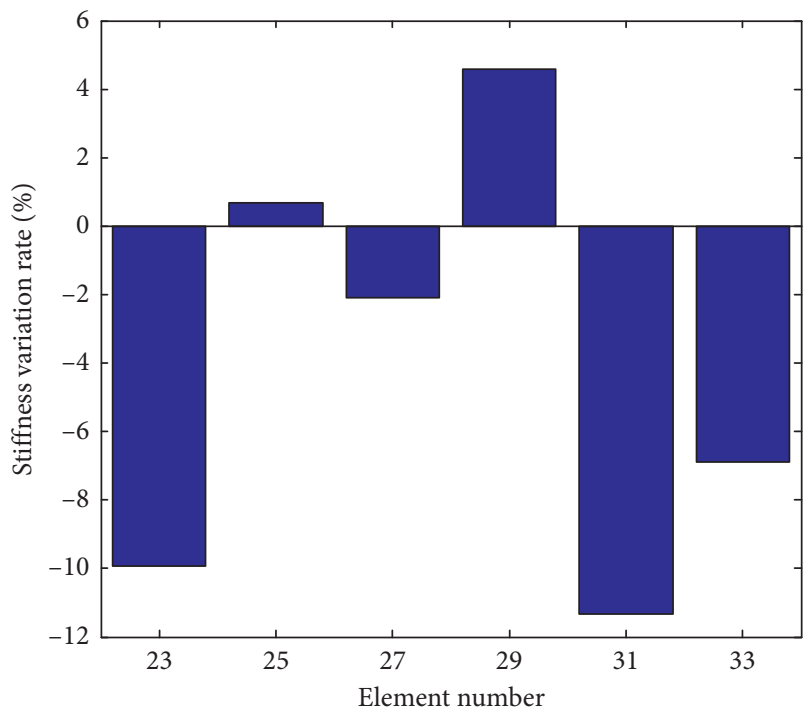

(d)

FIgURE 11: Continued. 


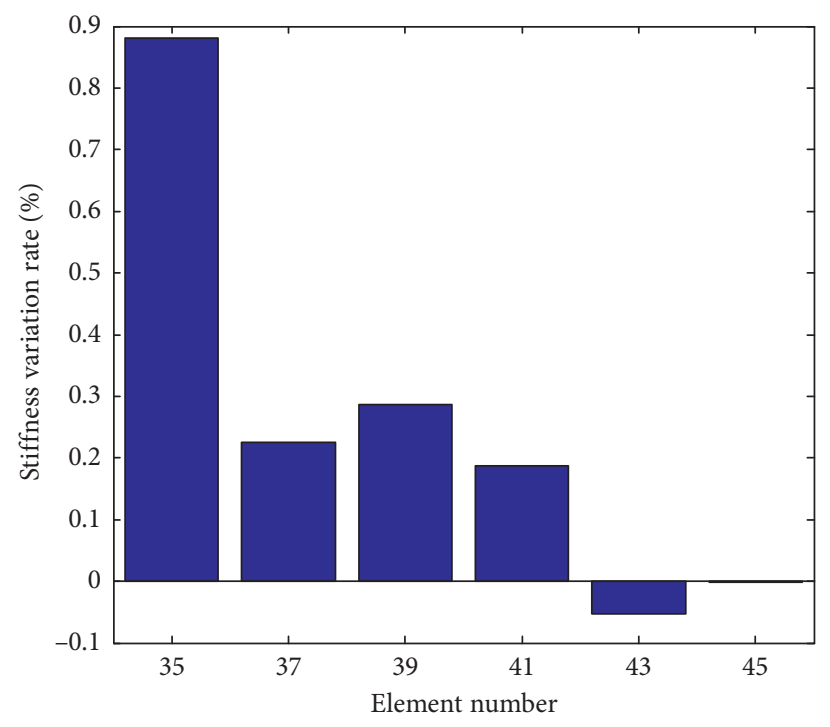

(e)

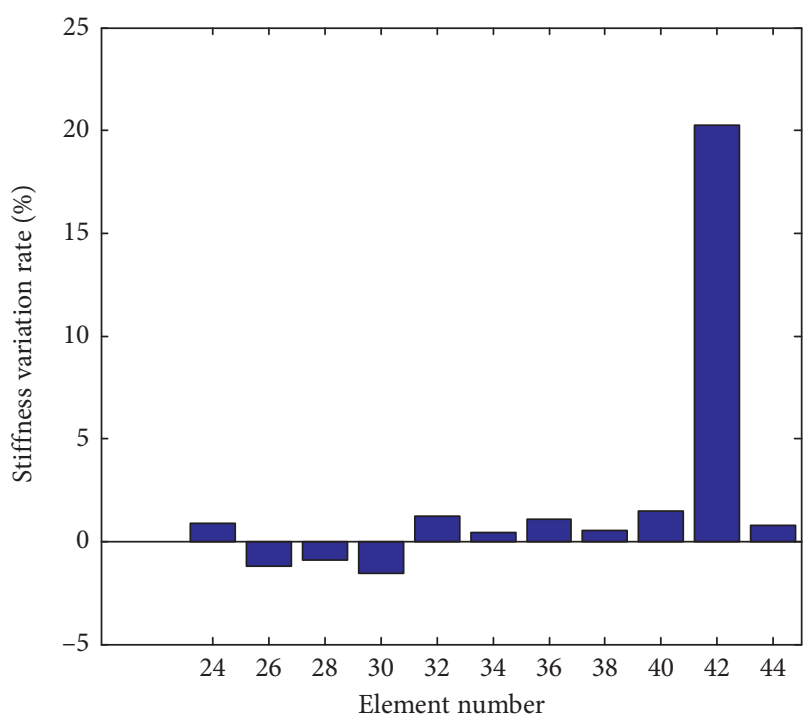

(f)

Figure 11: Damage detection using the expanded data contaminated by $1 \%$ noise: (a) displacement variations; (b) stiffness variation rate at lower chords; (c) stiffness variation rate at upper chords; (d) stiffness variation rate at diagonal elements; (e) stiffness variation rate at diagonal elements; (f) stiffness variation rate at vertical elements. In (a), the solid line indicates the horizontal displacement and the dashed line indicates the vertical displacement.

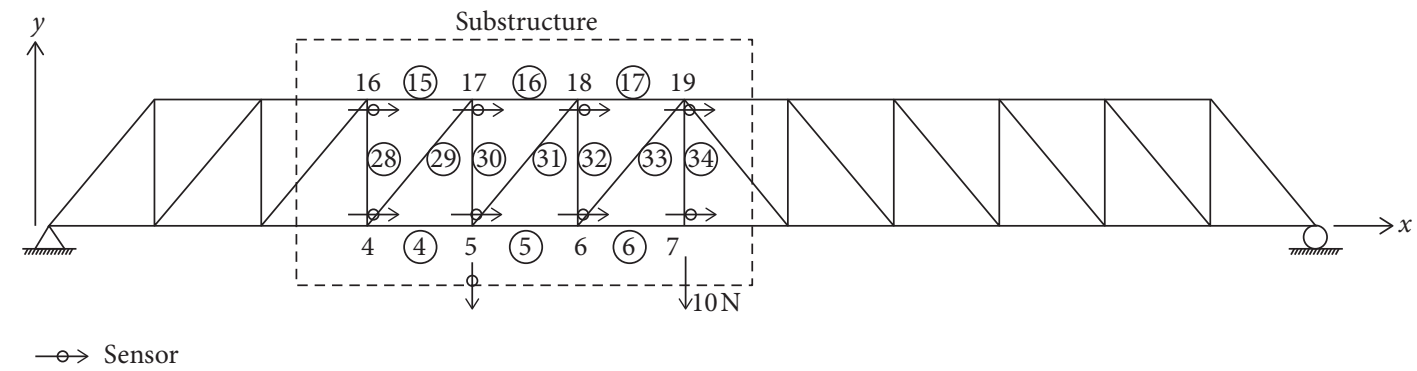

Figure 12: A substructure isolated from the entire truss structure.

cases demonstrate that the damage at elements 5 and 17 of the lower and upper chord members can be explicitly detected. Moreover, the diagonal and vertical elements 28, 29, and 30 display the same damage rate of $20 \%$, and it is believed that the damage is located at one of these. The damage of the diagonal and vertical members can be estimated by the installation and measurement of additional sensors. It is observed that the method is not sensitive to noise from the plots. The large stiffness variation rate at elements 33 and 34 is owing to the external load because these are adjacent to the loading point.

2.2.4. Dynamic Substructuring Approach. The dynamic approach begins with the measured mode shape data, as with the displacements in the static approach. The stiffness variation rate is calculated by the mode shape data. This work uses the complete or incomplete mode shape data corresponding to the first natural frequency only. The incomplete mode shape data are expanded by equation (3) with the stiffness matrix in the static approach. The damage detection on the truss structure in Figure 12 is performed by means of the substructuring method. The sensor locations are the same as in the previous example. In the noise-free case illustrated in Figure 14(a), the damage at the lower and upper chord members of 5 and 17 can be explicitly detected by the abrupt stiffness variation rate. The stiffness variation rates do not coincide with the damage rate because the analysis considers the first mode only and neglects the others. The damage at diagonal member 29 is not definitely detected because the adjacent members 28, 29, and 30 exhibit similar stiffness variations. The inadequacy of the numerical results arises from the neglect of the other modes. Similar results are indicated in the $5 \%$ noise case illustrated in Figure 14(b). It is observed that the damage at the vertical or diagonal members cannot be explicitly detected, but the damage-expected region can be predicted. The plots exhibit large variation rates at the members adjacent to the loading point. The variation rates near the loading point should be carefully investigated. 


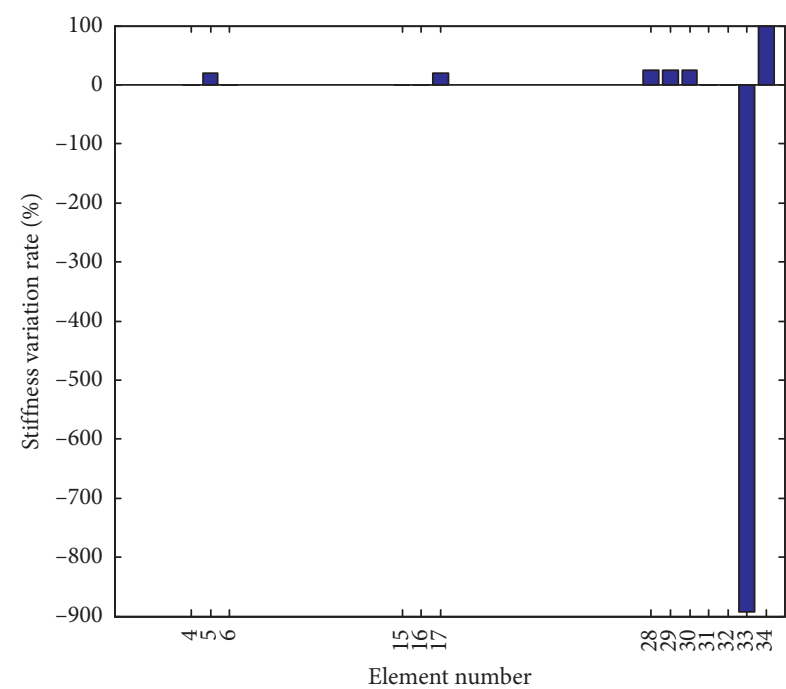

(a)

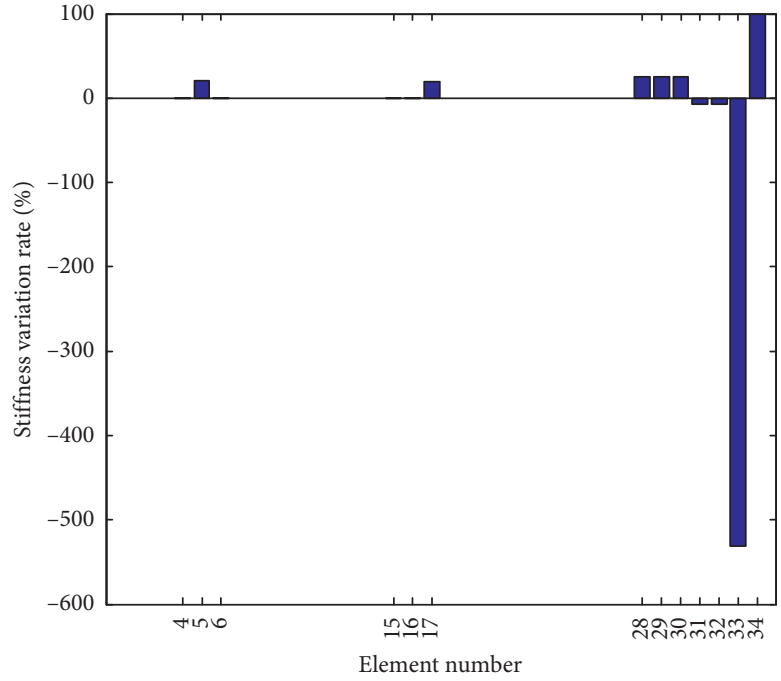

(b)

FIGURE 13: Damage detection by the substructuring method: (a) noise-free measurement data; (b) measurement data contaminated by $5 \%$ noise.

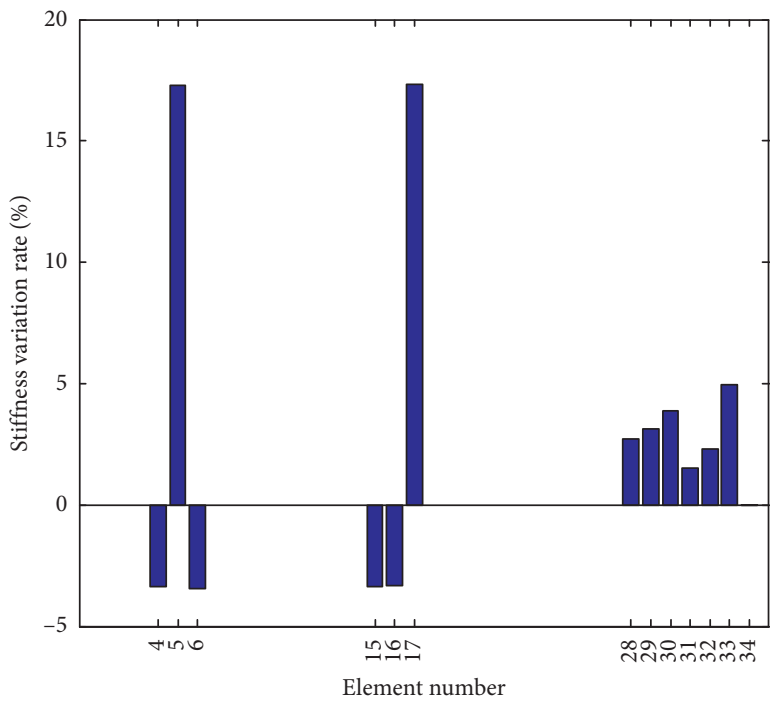

(a)

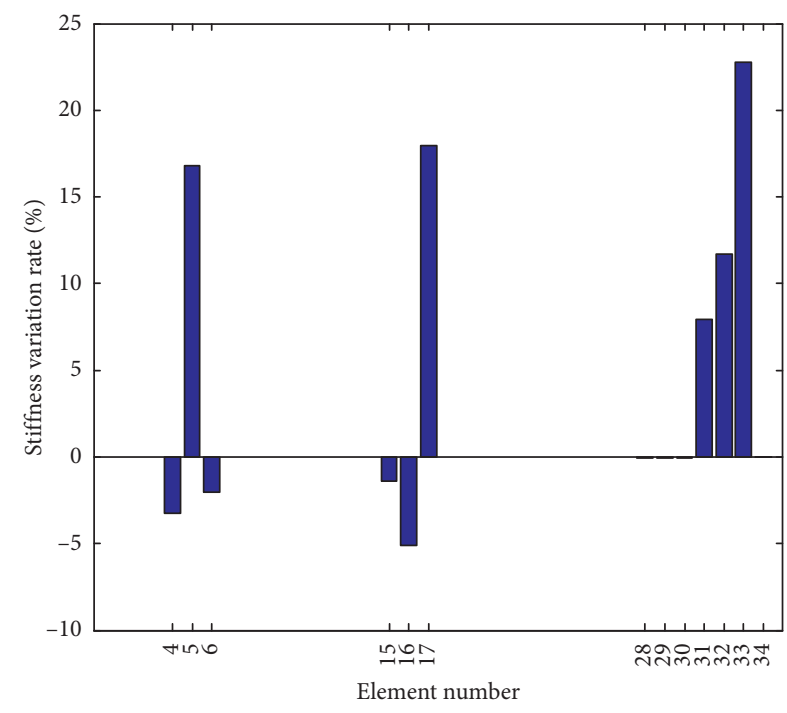

(b)

FigURe 14: Dynamic substructuring approach: (a) noise-free measurement data; (b) measurement data contaminated by $5 \%$ noise.

According to the static-based and dynamic-based examples, it is demonstrated that the stiffness variation rate calculated by the complete and accurate displacement data indicates the accurate damage locations, despite external noise. In the case that the number of measurement sensors is fewer than the system order, the expanded dataset provides information for predicting the damage region, including the damage-expected elements. The static-based and dynamicbased damage identification approaches by means of substructuring can detect the damaged elements more clearly, despite few measurement sensors and external noise. However, the damage of the diagonal and vertical members can be predicted by the member group. The damage- expected region should be investigated more thoroughly by means of the installation and measurement of additional sensors.

\section{Conclusions}

This work has investigated damage detection methods depending on the number of measurement locations to be causable in the evaluation of structural performance. This study has considered three different damage detection approaches utilizing complete and incomplete measurement data in an entire structure, and incomplete measurement data in substructures were compared. Furthermore, the 
noise effect contained in the measurement data was investigated. The measured and expanded displacements were transformed into the internal stress of the elements, and the stiffness variation rate was used as a damage index for evaluating the damage. The complete measurement data method exhibits the drawback of requiring the same number of sensors as the system order. The incomplete measurement data method exhibits the disadvantage of unstable data expansion depending on the number and installation locations of sensors. The substructuring method offers merits in reducing the system order and number of measurement sensors and is insensitive to external noise. However, the substructuring method needs to investigate the damageexpected region more closely for accurate damage detection by means of the installation and measurement of additional sensors.

\section{Data Availability}

The data used to support the findings of this study are available from the corresponding author upon request.

\section{Conflicts of Interest}

The authors declare that they have no conflicts of interest.

\section{Acknowledgments}

This research was supported by Basic Science Research Program through the National Research Foundation of Korea (NRF) funded by the Ministry of Education (NRF2016R1D1A1A09918011).

\section{References}

[1] B. Blachowski, Y. An, and B. F. Spensor Jr., "Substructurelevel based method for damage quantification in determinant trusses," in Proceedings of ISMA2016 Conference: International Conference on Noise and Vibration Engineering, Leuven, Belgium, September 2016.

[2] K. C. Park and G. W. Reich, "Model-based health monitoring of structural systems: process, potential challenges," in Proceedings of the 2nd International Workshop on Structural Health Monitoring, Stanford, CA, USA, September 1999.

[3] Z. Xing and A. Mita, "A substructure approach to local damage detection of shear structure," Structural Control Health Monitoring, vol. 19, pp. 309-318, 2012.

[4] U. Ugalde, J. Anduaga, F. Martinez, and A. Iturrospe, "SHM method for damage localization based on substructuring and VARX models," 2015, http://arxiv.org/abs/1501.01905.

[5] J. Hou, L. Jankowski, and J. Ou, "Substructure isolation and damage identification using free responses," Science China Technological Sciences, vol. 57, no. 9, pp. 1698-1706, 2014.

[6] Y. Lei, C. Liu, Y. Q. Jiang, and Y. K. Mao, "Substructure based structural damage detection with limited input and output measurements," Smart Structures and Systems, vol. 12, no. 6, pp. 619-640, 2013.

[7] E. T. Lee and H. C. Eun, "A model-based substructuring method for local damage detection of structure," Shock and Vibration, vol. 2014, Article ID 390769, 11 pages, 2014.

[8] J. Li, S. S. Law, and Y. Ding, "Substructure damage identification based on response reconstruction in frequency domain and model updating," Engineering Structures, vol. 41, pp. 270-284, 2012.

[9] Y. An, J. Ou, J. Li, and B. F. Spencer Jr., "Stochastic DLV method for steel truss structures: simulation and experiment," Smart Structures and Systems, vol. 14, no. 2, pp. 105-128, 2014.

[10] Y. An, B. Li, and J. Ou, "An algorithm for damage localization in steel truss structures: numerucal simulation and experimental validation," Journal of Intelligent Material Systems and Structures, vol. 24, no. 14, pp. 1683-1698, 2013.

[11] Q. W. Yang and W. M. Jin, "Damage detection for truss structures using incomplete modes," in Second International Workshop on Computer Science and Engineering, Bertinoro, Italy, November 2009.

[12] A. Kaveh and A. Zolghadr, "An improved CSS for damage detection of truss structures using changes in natural frequencies and mode shapes," Advances in Engineering Software, vol. 80, pp. 93-100, 2015.

[13] Z. D. Xu and K. Y. Wu, "Damage detection for space truss structre based on strain mode under ambient excitation," Journal of Engineering Mechanics ASCE, vol. 138, no. 10, pp. 1215-1223, 2012.

[14] M. Artar and A. Daloğlu, "Damage detection in simulated space frames using genetic algorithms," Sigma Journal of Engineering And Natural Sciences, vol. 33, no. 2, pp. 166-187, 2015.

[15] H. C. Eun, E. T. Lee, and H. S. Chung, "On the static analysis of constrained structural systems," Canadian Journal of Civil Engineering, vol. 31, no. 6, pp. 1119-1122, 2004. 


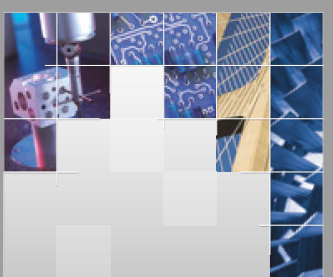

\section{Enfincering}
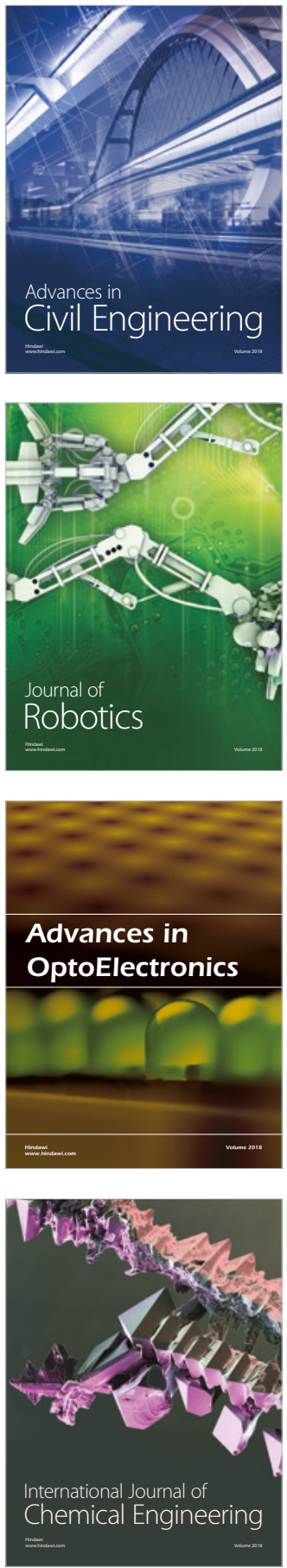

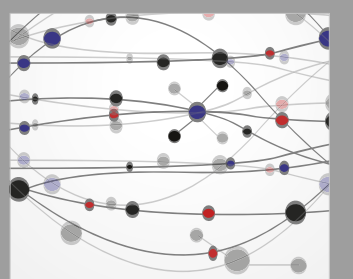

\section{Rotating \\ Machinery}

The Scientific World Journal

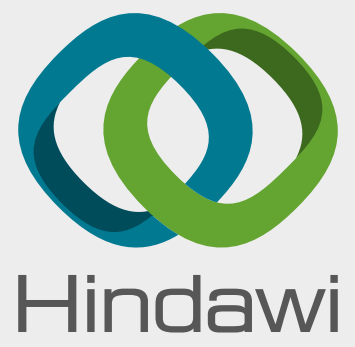

Submit your manuscripts at

www.hindawi.com
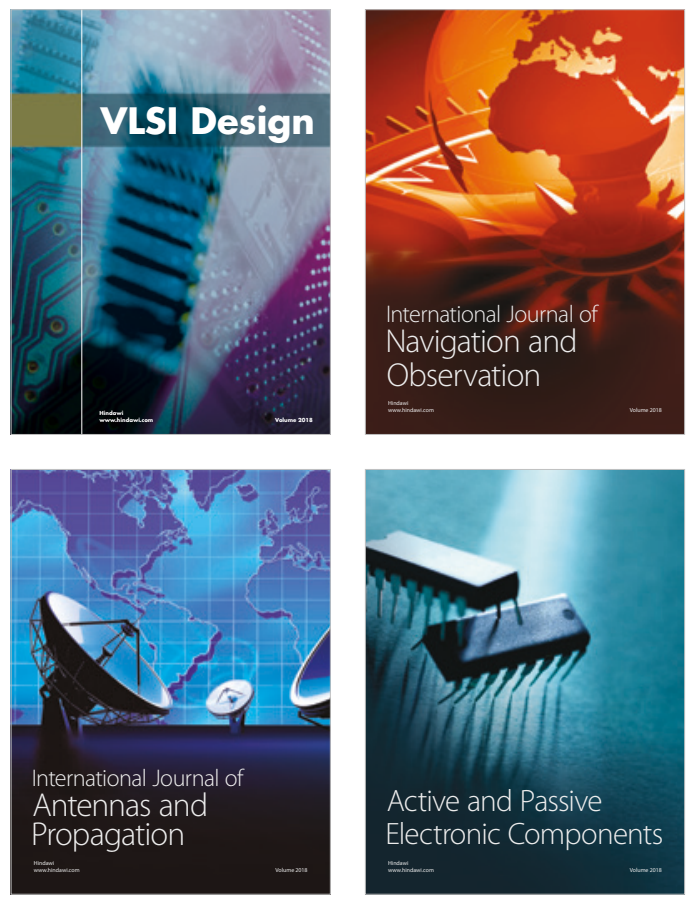
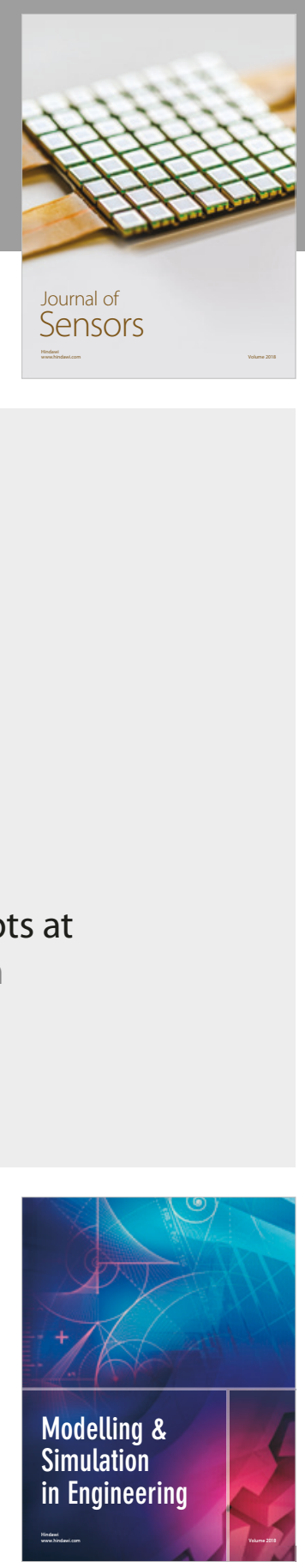

\section{Advances \\ Multimedia}
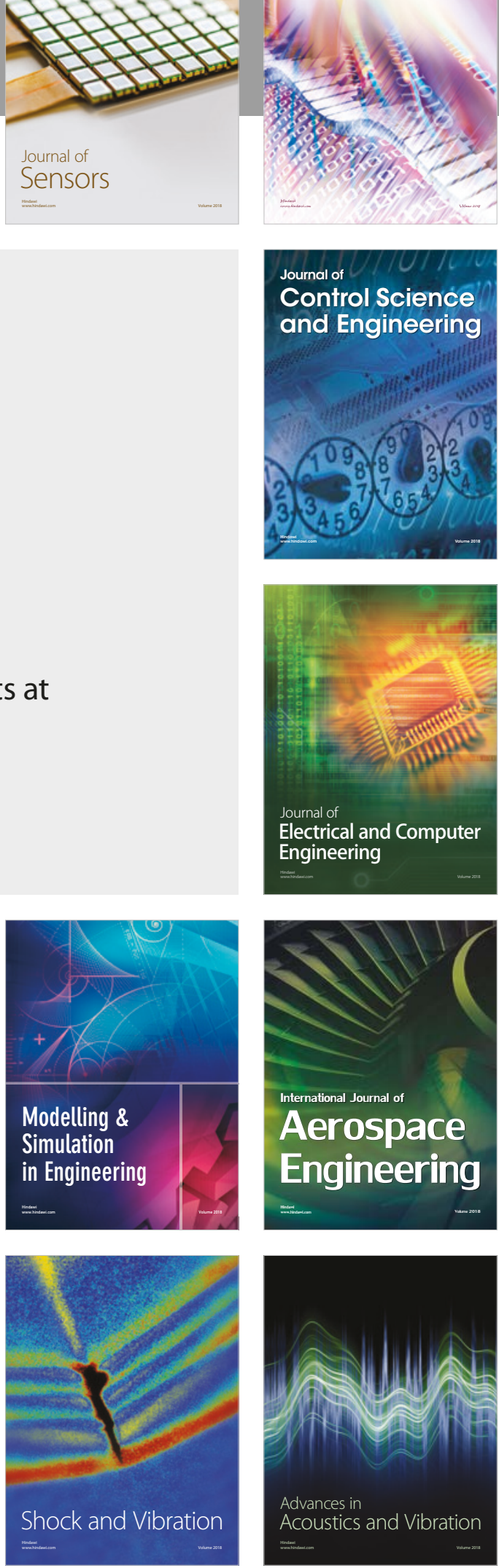